\title{
Una faglia tra storia e memoria. II centro di documentazione sul terremoto dell'Emilia.
}

L'articolo è un resoconto di un'esperienza di stage realizzata all'interno del Master di II livello in Public History di Unimore, in collaborazione con il Comune di Mirandola e l'Istituto Storico di Modena. La ricerca si è concentrata sull'avvio del futuro Centro di documentazione sul terremoto dell'Emilia.

The article is a report about a stage experience made whithin the Unimore Master's degree in Public History, in collaboration with Comune of Mirandola and Istituto Storico of Modena. The research has focused on the future documentation centre on Emilia earthquake.

\section{Introduzione}

È necessario collocare questo intervento nell'ottica della Public History. Quest'ultima si configura come una modo nuovo di fare Storia, e, volendo sintetizzare alcuni punti fermi, possiamo dire che l'obiettivo della Public History è quello di far uscire la storia dall'accademia; ha come destinatario il pubblico in generale al fuori delle università; si deve avvalere di numerosi e vari strumenti, tutti quelli a disposizione; ha come soggetto promotore uno storico di professione in collaborazione con esperti con altre competenze. In particolare, essa ha tra le proprie prerogative il fare storia vicino alle comunità sociali e il ricostruire memorie e identità su scale differenti, i principali ambiti di riferimento del presente lavoro. Dall'anno accademico 2015/2016, la Public History è diventata oggetto di un 
master universitario di II livello presso l'Università degli studi di Modena e Reggio Emilia. In questa cornice si presenta il futuro Centro di documentazione sul terremoto dell'Emilia, a cui è stato dedicato uno degli stage previsti dal piano di studi, in collaborazione con l'Istituto storico di Modena.

\section{L'oggetto}

Il centro di documentazione è espressione della volontà dell'amministrazione comunale della città di Mirandola e ha come obiettivo quello di raccogliere documenti, video e immagini relativi ai terremoti che hanno colpito la Bassa Modenese nel maggio del 2012, comprendendo i primi mesi di gestione dell'emergenza e gli anni di ricostruzione. Tutto il materiale andrà a confluire in uno spazio fisico appositamente creato e sarà a disposizione di studiosi, ricercatori, tecnici e di tutta la cittadinanza.

Il sisma è stato un evento di grande impatto sul piano materiale, culturale ed emotivo, è andato a segnare la storia e la memoria di una comunità e di un territorio, i quali non si aspettavano di poter vivere un evento del genere, poiché quel territorio, nel senso comune, non era percepito come ad elevato rischio sismico. Un territorio fortemente urbanizzato, per quanto decentrato rispetto alle città capoluogo, industrializzato e coltivato, con una efficace rete di trasporti e servizi, di punto in bianco si è ritrovata senza niente di funzionante. Qualsiasi attività era bloccata. La ripartenza c'è stata, ma ciò ha determinato un punto di rottura.

Questo centro di documentazione è un progetto monumentale che vede numerosi stakeholder. Il primo, ma solo perché ha lanciato il progetto, è il Comune di Mirandola. A cascata ne emergono altri: tante comunità, grandi e piccole, istituzionali e informali, adulti e bambini. In quanto evento "totale", il terremoto porta con sé una grande quantità di portatori di interesse. Parteciperanno comuni, regioni, fondazioni, aziende, associazioni professionali o di volontariato, fino a singoli cittadini, non solo del territorio, ma anche coloro che, pur abitando lontano, si sono spostati per portare aiuto. In questo caso, non sarà il public historian a portare la storia al pubblico, perché l'evento storico in questione è ben conosciuto dalla cittadinanza, non è un evento terzo rispetto ai due soggetti principali (lo storico e il pubblico). Perciò il public historian si presenterà come l'esperto che costruirà il discorso insieme alle comunità, attraverso un processo lento, che comporterà parecchio lavoro sul campo: parlare, intervistare, registrare, leggere, mettere in relazione, avanzare ipotesi e interpretazioni. Un vero lavoro da storico, fatto insieme ai soggetti ai quali il lavoro finale è destinato. 


\section{Documenti}

La raccolta delle fonti relative al terremoto dell'Emilia del 2012 è cominciata nel gennaio 2016, costituendo per lo più una mappatura delle potenziali direzioni di ricerca da seguire. Come prima cosa si è cercato di capire quali fossero gli enti e le persone che, in quei mesi o in questi anni, si siano occupati di gestire questo evento, che tipo di ruolo avessero e per quanto tempo è durato il loro impegno. Poi si è passati a una previsione della qualità del materiale da essi prodotto, ma soprattutto della quantità. Questo è un punto molto importante ai fini della ricerca, perché potrebbe sorgere il problema di avere troppe fonti, le quali rimarrebbero mute. Esistono diversi enti che hanno già archiviato nei loro uffici molto materiale, prettamente tecnico: non avrebbe senso riprodurlo anche nel centro di documentazione. Per fare alcuni esempi, presso le sedi della Regione Emilia Romagna si trovano archiviate tutte le schede Aedes per l'agibilità degli edifici; la sezione bolognese dell'Istituto nazionale di geofisica e vulcanologia (Ingv) ha raccolto migliaia di fotografie nelle prime settimane successive alle scosse principali; il sito del Comune di Mirandola raccoglie tutte le delibere approvate. In questi casi, il centro di documentazione dovrà fornire da spazio, fisico e digitale, che possa indirizzare l'utente verso ulteriori luoghi dove andare a cercare. Ma questo solo nel caso in cui ci sia un soggetto o un ente di grandi dimensioni che ha già operato una sintesi della propria azione, che raccoglie materiale strettamente tecnico. Oltre a questo, la mappatura ha raccolto altra documentazione, dalle tesi di laurea a colloqui informali che bisognerebbe trasformare in video-interviste, da resoconti di missione a scritti privati, da esperienze scolastiche a pubblicazioni della Regione Emilia-Romagna, prodotte nei mesi successivi all'evento. L'orizzonte geografico è rimasto inizialmente legato a Mirandola, perché, fino a ora, interpretazioni generali e concluse di aspetti della gestione dell'emergenza non ne sono stati fatti, se non dall'azienda sanitaria locale. Inoltre, si sta compilando anche una bibliografia sul terremoto del 2012, su precedenti terremoti italiani e sui fenomeni sismici in generale, in vista di una potenziale sezione dedicata all'interno del centro di documentazione, che raccolga non solo saggi, ma anche testimonianze, racconti, romanzi, graphic novel, poesie.

Qui di seguito presentiamo una selezione di fonti significative raccolte fino a oggi.

Il primo è un volantino sgualcito e calpestato, datato 20 maggio 2012, con appuntate sul retro, in un corsivo frettoloso, alcune precise indicazioni: «Rimanere fuori fino alle 11 tenendosi in posizione di sicurezza. Lasciare libera le strade per i mezzi di soccorso». Il messaggio venne ripetuto con il megafono girando per 
le strade del paese, durante una mattina non facile da affrontare. Questo pezzo di carta, custodito gelosamente dal Sindaco di Mirandola nel proprio ufficio, restituisce il senso profondo dell'iniziativa di avviare un centro di documentazione sul terremoto: un foglietto qualunque, raccolto da terra, che avrebbe potuto essere buttato, scordato, dimenticato, e invece è stato custodito perché rappresentava un momento inedito nella vita della comunità mirandolese.

Importantissimo per cominciare la ricerca è stato il faldone con tutte le richieste di accesso alla zona rossa del centro storico di Mirandola, avanzate tra la fine di maggio e agosto del 2012. A partire da questo si è potuto, prima di tutto, fare una panoramica generale di chi fosse interessato a entrare in quella parte del centro abitato, per quale motivo e da dove provenisse: ricercatori, fotografi, giornalisti e testate televisive, sia italiani sia stranieri. Un ulteriore filone di fonti interessanti è quello del materiale prodotto dal mondo della scuola e dell'università, una prova

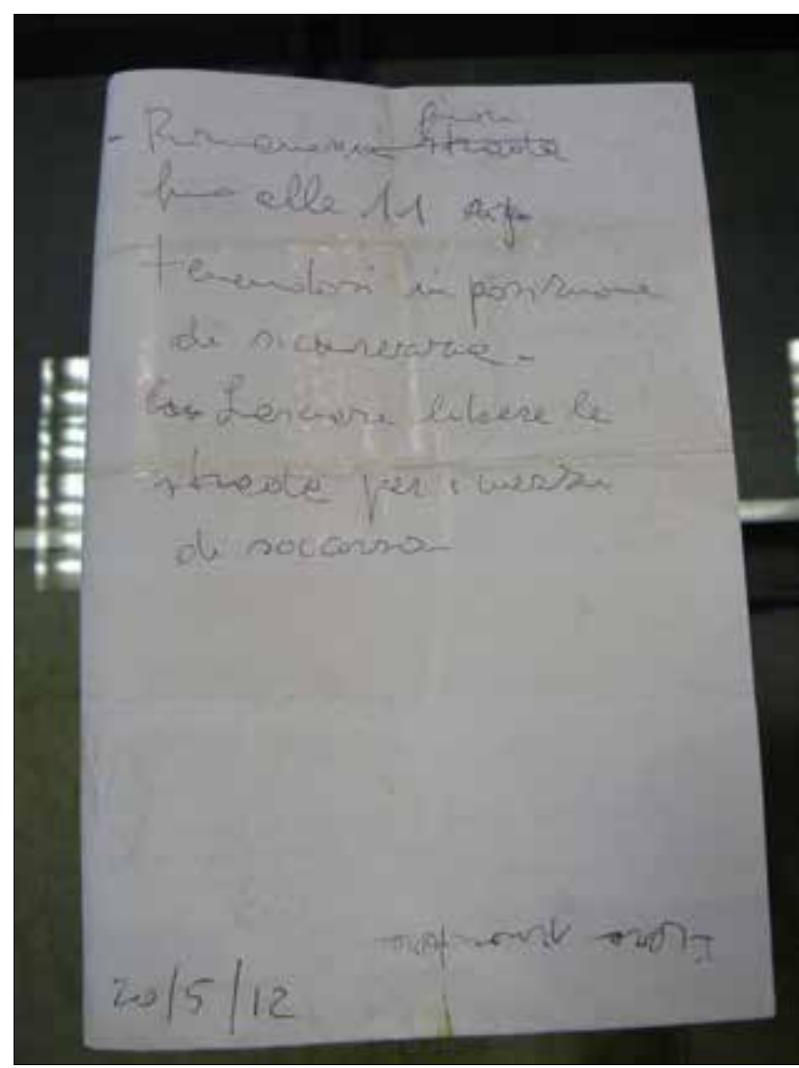

Fig. 1. Il volantino del 20 maggio 2012, custodito dal Sindaco di Mirandola. del bisogno di riflessione e di rielaborazione su un'esperienza particolarmente intensa e inedita come un terremoto. Vediamo nelle seguenti immagini la fotocopia di un tema scolastico, i dvd di una canzone e di un telegiornale scolastico realizzati dagli studenti della scuola secondaria di primo grado "Montanari", due delle numerose tesi di laurea che studenti universitari hanno dedicato all'argomento.

Dopo la pubblicazione degli avvisi pubblici, sono giunti in Comune anche fonti memorialistiche, nate dall'esigenza di mettere su carta quanto vissuto, provato e sofferto tra maggio e giugno del 2012. In prosa o in poesia, ecco che emerge uno 


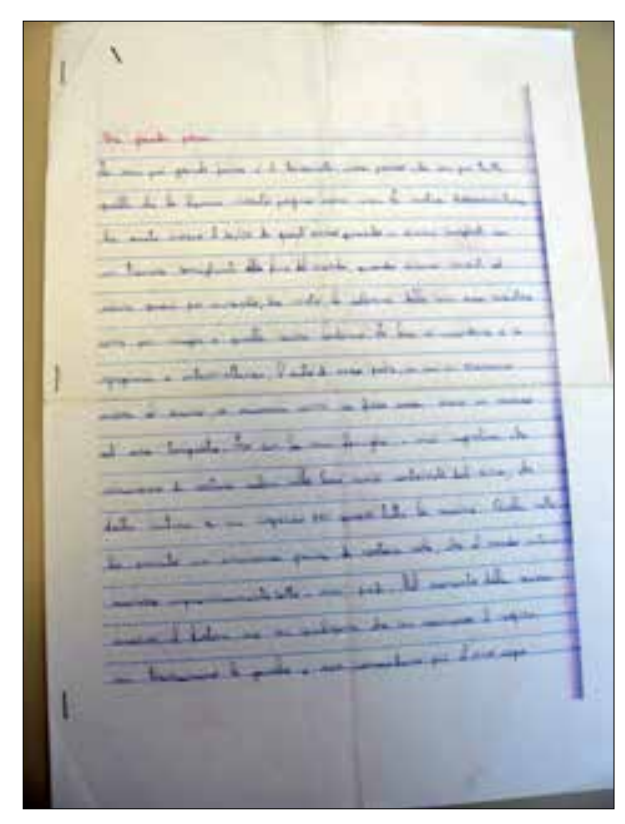

Fig. 2. Tema scolastico.

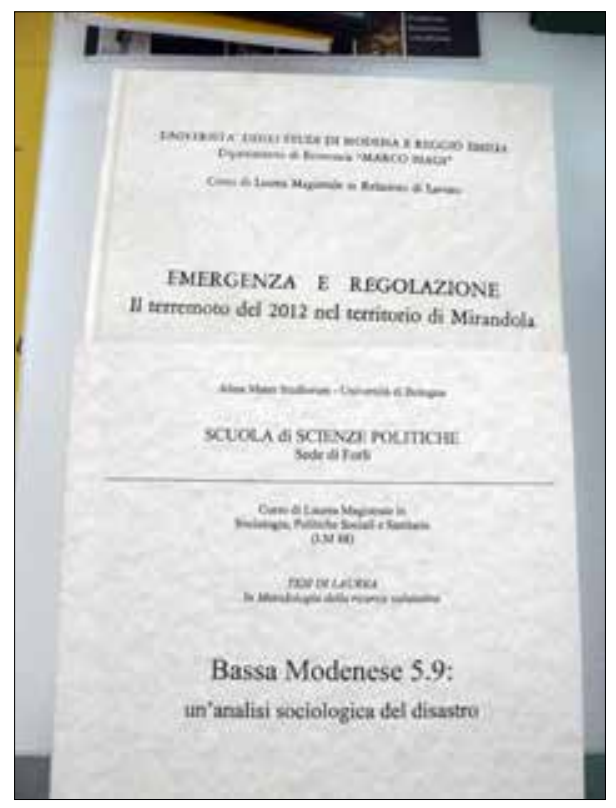

Fig. 5. Due tesi di laurea sul sisma del 2012.

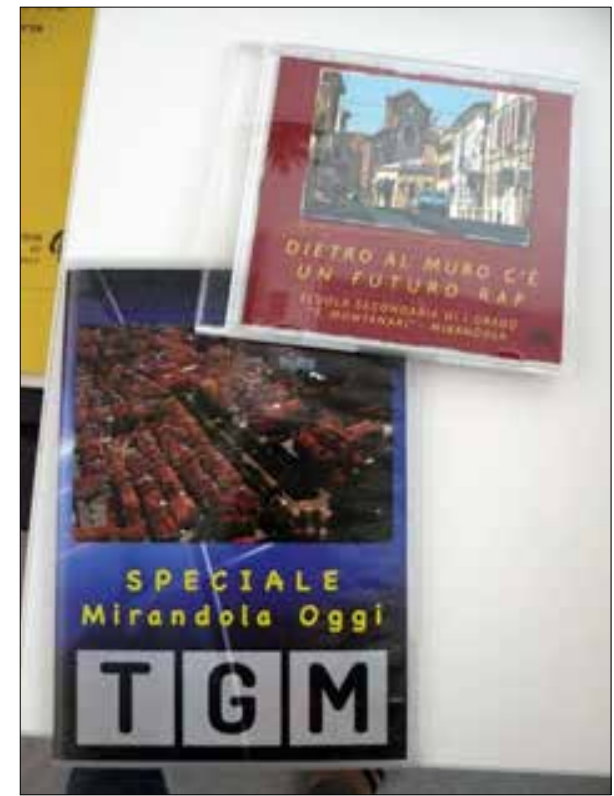

Fig. 3. Progetti realizzati dagli studenti della scuola "Montanari" di Mirandola.

dei ruoli fondamentali della scrittura, quello di dare forma al nostro vivere interiore. Si tratta di privati cittadini, alcuni anche molto anziani, che con apparente lucidità raccontano momenti sicuramente non facili da vivere, ma da cui traspare un forte senso di comunità. Un altro tipo di materiale documentario è fornito dalle pubblicazioni ufficiali prodotte in particolare dalla Regione Emilia Romagna: testi sull'edilizia scolastica, sulla reazione del distretto biomedicale, sugli edifici ricostruiti grazie alle donazioni, sull'ambito geologico e della rilevazione di agibilità. Infine, è stata avviata anche una raccolta di fonti bibliografiche sui vari aspetti del sisma e sui terremoti italiani, che permettono di fare profonde riflessioni e confronti sul tema. Tra i titoli possiamo citare Terremoto dentro [Pederzoli 2013], una raccolta di scritti all'interno di un 


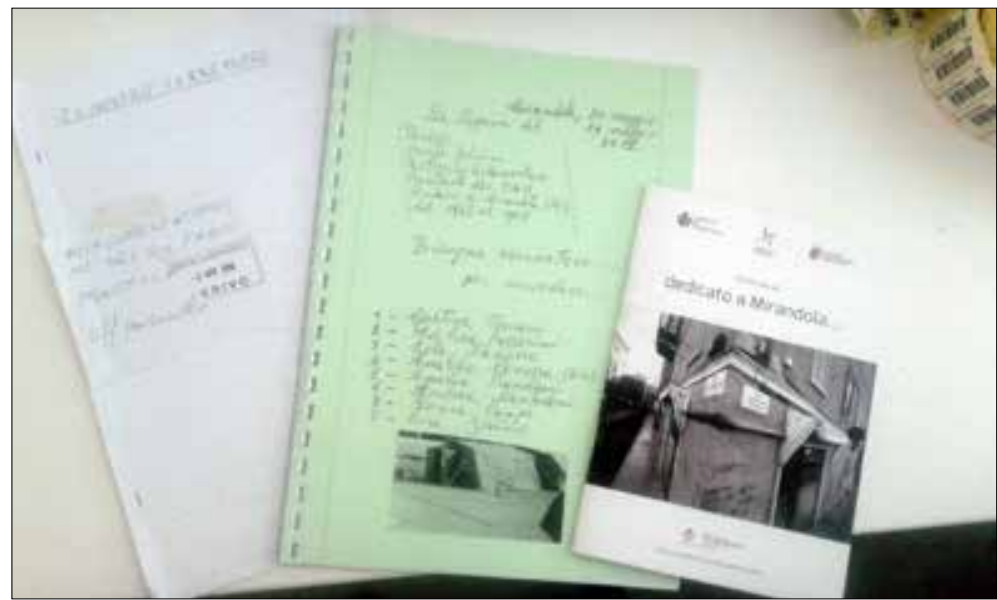

Fig. 5. Raccolta di scritti privati, tra cui un opuscolo firmato da "Le Signore del parco Oreste Gelmini”.

laboratorio di scrittura in seguito al terremoto dell'Aquila; la pubblicazione del centro Belice/Epicentro in merito alla loro esperienza di museo nella zona di Gibellina [Maiorana 2016]; il dvd pubblicato dall'Ingv sulla memoria e i luoghi della memoria del terremoto del Belìce. Oppure si possono trovare titoli generali sui terremoti [Massa e Camassi 2013], sui terremoti dell'Italia unita [Guidoboni e Valenzise 2011], o di taglio scientifico interdisciplinare [Carnelli e Ventura 2015]. Per finire, non bisogna dimenticare le numerose registrazioni di preziose testimonianze orali.

\section{Prospettive}

Sarà pertanto necessario, dopo aver attribuito una peculiare identità a questo Centro di documentazione, inserirlo in una rete almeno a livello nazionale e, soprattutto, individuare il modo migliore per animarlo, trasformandolo in un reale centro di aggregazione e promozione culturale. Come ci insegna la sismologia storica, ogni terremoto fa storia a sé, avviene in uno specifico territorio e gli scenari che si determinano sono estremamente diversi. Il terremoto dell'Emilia è avvenuto come un fulmine a ciel sereno, poiché non c'era memoria di eventi analoghi tramandata fra le generazioni. È la prima testimonianza che riportano molte delle persone che lo hanno vissuto. Ed è un pensiero corretto e condivisibile, ma proprio perciò la memoria va salvaguardata e alimentata. Altrimenti, con il tempo questa sequenza sismica potrebbe diventare un ricordo lasciato alle spalle, e con esso probabilmente anche l'interesse per lo studio dell'evento e per il tema più generale del rischio sismico. Cosa fare, allora, se davvero la necessità della memoria e dello sviluppo di una cultura della prevenzione vogliono essere mantenuti? Quattro direzioni in particolare: 
- fare rete con altre esperienze in Italia, lavorando alla creazione di una cultura del terremoto e della prevenzione a livello nazionale, collettivizzando le proprie storie e le proprie strategie;

- farlo diventare un polo di ricerca, di studio, di innovazione sociale e culturale su questo tema, in stretta relazione con strutture che di terremoti si occupano attivamente, o ancora con le strutture universitarie, i musei, gli istituti storici e culturali;

- farlo diventare un polo di innovazione didattica, poiché è dalle giovani generazioni che bisogna partire, perché la cultura della prevenzione è un investimento sociale e politico sul futuro, che significa offrire occasioni di formazione e riflessione a tutti coloro che lavorano nelle agenzie educative del territorio, la scuola in particolare;

- farlo diventare un polo per le pratiche di solidarietà, che sia centro di aggiornamento permanente sulla tematiche della conoscenza e della prevenzione al rischio sismico, sfruttando, rafforzando e mettendo in rete il popolato mondo del volontariato.

Il centro di documentazione di Mirandola, proprio per la sua identità atipica di zona in cui è avvenuto un terremoto della cui possibilità era inconsapevole, dovrebbe farsi portatore di un'identità-collante con il resto d'Italia, paese a elevato rischio sismico e idrogeologico. Un primo passo verso questa direzione è stato il convegno Terre in movimento, svoltosi il 18 giugno presso la Sala consiliare del Comune di Mirandola. Scorrendo l'elenco dei relatori, emerge a prima vista il fatto che si è voluto fin da subito dare un'impostazione storica al dibattito: un incontro mai avvenuto prima tra diverse comunità che, da un capo all'altro del paese, hanno vissuto un terremoto nel corso degli ultimi decenni, ognuno con le sue specificità. Ruolo delle istituzioni, rapporti tra le istituzioni, ruolo e composizione della cittadinanza, numero e intervallo delle scosse, conformazione del territorio, sono tutti argomenti affrontati in una comparazione tra le esperienze stori-

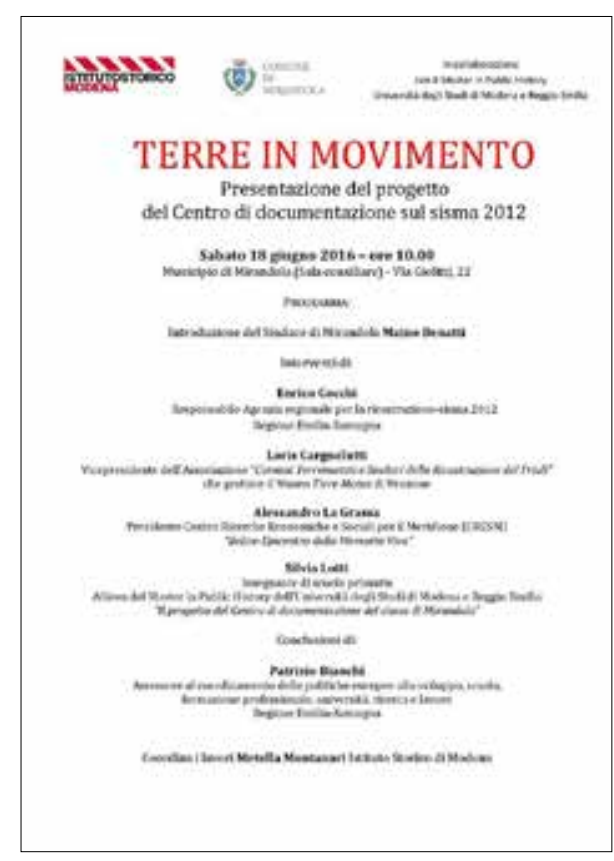

Fig.6: Locandina del convegno Terre in movimento. 
che. I recenti terremoti verificatisi tra Marche, Umbria, Lazio e Abruzzo non fanno altro che confermare la necessità di lavorare in modo collettivo per la costruzione di una cultura del terremoto e della prevenzione.

\section{Bibliografia}

Carnelli F. e Ventura S. 2015, Oltre il rischio sismico. Valutare, comunicare e decidere oggi, Roma: Carocci.

Guidoboni E. e Valenzise P. 2011, Il peso economico e sociale dei disastri sismici in Italia negli ultimi 150 anni, Bologna: Bononia University Press.

Maiorana G. 2016, Gibellina. Il museo da vivere, racconto di un'esperienza, Acireale: New'link.

Massa M. e Camassi R. 2013, I terremoti. Quando la terra trema, Bologna: il Mulino.

Pederzoli A. (ed.) 2013, Terremoto dentro, Bologna: Narcissus.

\section{Risorse on line}

Laboratorio Energie Sisma Emilia

http://www.energie.unimore.it/

Museo Tiere Motus di Venzone (UD)

http://www.tieremotus.it/

Museo Belìce/Epicentro della memoria viva

http://www.epicentrobelice.net/

Osservatorio sul dopo sisma

http://www.osservatoriosuldoposisma.com/

Laboratorio sismico Alta Valtiberina

https://www.facebook.com/Laboratorio-Sismico-alta-Valtiberina-885523421538238/ 